Documentation et bibliothèques

\title{
Les besoins et les habitudes documentaires des chercheurs des trois centrales syndicales au Québec
}

\section{Marc Comby}

Volume 38, numéro 3, juillet-septembre 1992

URI : https://id.erudit.org/iderudit/1028625ar

DOI : https://doi.org/10.7202/1028625ar

Aller au sommaire du numéro

Éditeur(s)

Association pour l'avancement des sciences et des techniques de la

documentation (ASTED)

ISSN

0315-2340 (imprimé)

2291-8949 (numérique)

Découvrir la revue

Citer ce document

Comby, M. (1992). Les besoins et les habitudes documentaires des chercheurs des trois centrales syndicales au Québec. Documentation et bibliothèques, 38(3), 163-168. https://doi.org/10.7202/1028625ar

Tous droits réservés (c) Association pour l'avancement des sciences et des techniques de la documentation (ASTED), 1992
Ce document est protégé par la loi sur le droit d'auteur. L'utilisation des services d’Érudit (y compris la reproduction) est assujettie à sa politique d'utilisation que vous pouvez consulter en ligne.

https://apropos.erudit.org/fr/usagers/politique-dutilisation/ 


\title{
Les besoins et les habitudes documentaires des chercheurs des trois centrales syndicales au Québec
}

\author{
Marc Comby* \\ archiviste \\ Syndicat des professeurs et professeures de l'UQAM \\ Montréal
}

Au Québec, les recherches provenant des milieux syndicaux sont plutôt méconnues. La quantité et la qualité de ces recherches y sont pourtant impressionnantes. Depuis nombres d'années, les centrales syndicales portent d'ailleurs un intérêt particulier au développement de leur centre de documentation.

Par notre recherche, nous avons voulu connaître deux réalités: 1) les types de recherche syndicale et les thèmes étudiés par les chercheurs sont-ils identiques d'une centrale à l'autre? 2) est-ce que, de façon générale, les besoins documentaires des chercheurs sont identiques d'une centrale à l'autre? Cette étude, qui porte sur les besoins et les habitudes documentaires des chercheurs syndicaux, aidera à mieux comprendre leurs besoins. Elle pourra, de plus, ouvrir des perspectives pour permettre, nous l'espérons, aux centres de documentation de mieux répondre aux besoins de ces chercheurs. La présente recherche brossera un tableau général des besoins et habitudes documentaires des chercheurs syndicaux. Ce tableau se divisera en quatre blocs: la provenance des demandes en matière de recherche et la diffusion des résultats de recherche; l'utilisation des sources documentaires écrites par les chercheurs; les sources orales d'information; l'utilisation des bases de données.

La méthode de cueillette de données a consisté en des entrevues personnelles sur la base d'un questionnaire pré-établi. La revue de la littérature nous a aidé à construire ce questionnaire.

Notre échantillon se limite aux grandes centrales syndicales, la Fédération des travailleurs du Québec (FTQ), la Confédération des syndicats nationaux (CSN) et la Centrale des enseignants du Québec (CEQ). Sept chercheurs ont été rencontrés, deux chercheurs de CEQ, deux de la FTQ et trois de la CSN.

\section{Petite histoire et contexte de la recherche syndicale}

Au Québec, les premières organisations syndicales sont apparues dans les années $1820^{1}$. Aujourd'hui, elles regroupent $65,3 \%$ des travailleurs dans le secteur public et $31 \%$ dans le secteur privé. La FTQ, la CSN et la CEQ regroupent, à elles trois, plus de $60 \%$ de tous les syndiqués au Québec ${ }^{2}$ provenant de tous les secteurs de la production (institutions bancaires, industries du meuble et de la construction, organismes publics, etc.).

La portée des interventions des centrales syndicales n'est pas strictement syndicale. Elle atteint les domaines économiques, sociaux, culturels et politiques. Nous voyons les syndicats se solidariser avec des travailleurs d'autres pays. Ils sont intervenus dans des questions d'enjeuxnationaux tels que le libre-échange, la question constitutionnelle, la langue française, etc. Les chercheurs sont ainsi amenés à faire des travaux sur des thèmes qui vont bien au-delà des affaires proprement syndicales. De tous temps, les syndicats ont ainsi produit des recherches dans divers domaines, que ce soit des analyses économiques, des prises de positions ou des études réalisées dans le but d'étoffer des cahiers de revendications.

Notons que la création de l'Institut de recherche appliquée sur le travail (IRAT) ${ }^{3}$ en 1972 et l'élaboration, en 1976, du Protocole d'entente UQAM-CSN-FTQ ${ }^{4}$ constituent un tournant marquant en faveur de la recherche syndicale au Québec et du soutien aux interventions syndicales.

\section{Revue de la littérature}

À travers la revue de la littérature, nous n'avons pas trouvé d'articles traitant des besoins des chercheurs syndicaux au Québec. Par contre, nous avons relevé deux mémoires de maîtrise qui portent respectivement, l'un sur les habitudes documentaires des recherchistes de trois réseaux montréalais de télévision de langue française, et l'autre sur les sources d'information et habitudes documentaires des journalistes de la presse quotidienne francophone du Québec.

Nicole Grimard, dans son étude sur les habitudes documentaires des recherchistes de la télévision ${ }^{5}$, émet 6 hypothèses divisées en deux blocs. Dans le premier, elle vérifie l'utilisation des services documentaires en fonction: de la formation; du temps disponible; des śervices offerts par les réseaux. Dans le deuxième

* Cet article fut à l'origine une recherche soumise dans le cadre d'un cours du programme de maîtrise «Recherche en communication de l'information», donné à l'École de bibliothéconomie et des sciences de l'information de l'Université de Montréal.

1. Jacques Rouillard, Histoire du syndicalisme québécois, Montréal, Boréal, 1989, p.15.

2. Le Québec statistique, Québec, Les publications du Québec, 1989, p.346. De ces pourcentages, il faut exclure le secteur de la construction et les conventions collectives de compétence fédérale.

3. L'Institut de recherche appliquée sur le travail est une société sans but lucratif. Il a été fondé conjointement par les trois centrales syndicales.

4. Le Protocole est défini comme un «guichet d'accès aux ressources de l'UQAM». Des professeurs réguliers sont dégrevés de leurs charges d'enseignement pour réaliser des recherches au bénéfice des centrales. Les demandes en matière de recherche proviennent principalement de la FTQ et de la CSN.

5. Nicole Grimard, Les habitudes documentaires des recherchistes de trois réseaux montréalais de télévision de langue française, mémoire de maîtrise déposé à l'École de bibliothéconomie et des sciences de l'information de l'Université de Montréal, 1988. 
bloc, elle vérifie la perception de l'utilité des sources documentaires en fonction: de la formation; du temps disponible; des services offerts par les réseaux. Sans énumérer les nombreux résultats de sa conclusion, mentionnons les observations suivantes: «Plus la qualité des services offerts est grande, plus l'utilisation qui en est faite est élevéen". Jean de Bonville, de son côté, a analysé les sources d'information utilisées par les journalistes de la presse quotidienne francophone et a décelé leurs habitudes documentaires ${ }^{7}$. Les deux études de N. Grimard et de J. de Bonville nous ont été utiles pour élaborer notre questionnaire et faire le meilleur choix méthodologique pour la cueillette des données.

Dans une autre étude, Marie-France Rochard a réalisé une enquête auprès des chercheurs scientifiques d'une université à Lyon ${ }^{8}$. Devant la diminution des fonds documentaires dans les bibliothèques scientifiques à Lyon, M.-F. Rochard s'est demandée s'il était essentiel de maintenir certains abonnements de périodiques alors que les chercheurs peuvent se procurer les articles par d'autres voies? L'auteure a analysé les habitudes documentaires des chercheurs scientifiques à l'aide d'un questionnaire et elle termine son étude en suggérant la création d'un «système d'organisation documentaire favorable aux chercheurs»" ${ }^{9}$ qui serait moins coûteux pourl'Université. L'Article deM.-F. Rochard nous a permis de réfléchir sur les moyens à prendre pour sauvegarder les services documentaires dans un contexte de hausse des coûts et de diminution des budgets d'acquisition.

Mary B. Folster a étudié, dans une université américaine, les différences entre les besoins d'information des chercheurs de faculté, les étudiants gradués qui ont complété les examens préliminaires et les étudiants qui n'ont pas complété les examens préliminaires ${ }^{10}$. Elle conclut que les trois groupes consultent sensiblement les mêmes sources d'information. L'importance de chaque source, par contre, diffère selon les groupes.

La revue de la littérature nous a fait aussi connaître les rapports entre les services d'éducation ouvrière et les bibliothèques publiques aux États-Unis. Martha Jane Soltow fait un survol historique des services fournis par les bibliothèques publiques aux organisations de travailleurs ${ }^{11}$. Dès les années 1920 aux États-Unis, les bibliothécaires reconnaissaient les besoins spécifiques des travailleurs. C'est surtout après la Seconde guerre que les rapports entre les bibliothèques et les organisations se transforment avec la création du Comité conjoint AFL-CIO/ALA sur les services de bibliothèques aux groupes de travailleurs.

\section{Profil des chercheurs}

Les chercheurs rencontrés en entrevue ont tous une scolarité poussée. Cinq d'entre eux possèdent une maîtrise ou un doctorat tandis que les deux autres ont d'autres diplômes. Les chercheurs ont en moyenne plus de 10 ans d'expérience en recherche. Dans chacun de leur syndicat respectif, les chercheurs ont plus de 6 années d'expérience dans leur emploi.

\section{Un aperçu des centres de documenta- tion syndicaux}

Le centre de documentation de la CSN est informatisé. II regroupe une collection d'ouvrages de référence généraux et spécialisés, une centaine de périodiques, les publications de la centrale, des publications des gouvernements du Québec et du Canada, des publications d'organismes québécois et canadiens qui oeuvrent dans les secteurs communautaires, universitaires, syndicaux et politiques et finalement des publications d'organisations internationales. De plus, la CSN est abonnée à un nombre de serveurs (Dialog, SDM, CAN/ OLE, INFO-GLOBE, FP ONLINE, INFOMAT ONLINE, KNOWLEDGE IN$D E X)$ ainsi qu'à un courrier électronique (ENVOY 100). Il faut aussi préciser que la totalité de la documentation n'est pas regroupée au centre de documentation. Le Service de recherche possède aussi sa propre documentation et est abonné à ses propres serveurs.

À la CEQ, le centre de documentation est aussi informatisé. La base de données du catalogue du centre se trouve chez le serveur Services documentaires multimedia inc. (SDM) suite à une entente de services entre les deux parties. L'indexation, la saisie et la correction des bordereaux sont faites au centre de documentation de la centrale. Les notices traitées sont ensuite télédéchargées dans la base de données chez SDM. Seuls les membres de la CEQ ont accès à la base. Occasionnellement, les usagers extérieurs peuvent la consulter après autorisation. Selon les besoins des chercheurs, à chaque année, une partie de la base de données est convertie en micro-fiches. Par ailleurs, des données peuvent être, en tout temps, importées de SDM et gérées dans la base de données locale du centre de documentation (logiciel «Naturel»). Le centre est abonné à environ 300 périodiques et possède un fonds documentaire d'environ 10000 monographies. Les types de publications conservées au centre sont sensiblement les mêmes qu'à la CSN. Le centre offre, en plus, un service de presse où sont dépouillés les principaux quotidiens ( $L a$ Presse, Le Devoir et Le Soleil). Dans le cas des quotidiens The Gazette et Le Journal de Montréal, les attachés de presse de ces journaux font parvenir au centre de documentation les coupures de presse qui concernent la CEQ. Dans les régions, les syndicats locauxfont aussi parvenir des coupures de leurs quotidiens respectifs (par exemple, Le Nouvelliste à Trois-Rivières). Finalement, le centre a accès aux principaux serveurs (SDM, DIALOG, QUESTEL, DOBIS).

6. Ibid., p.149.

7. Jean de Bonville, Sources d'information et habitudes documentaires desjournalistes de lapresse quotidienne francophone du Québec, mémoire de maîtrise déposé à l'École de bibliothéconomie et des sciences de l'information de l'Université de Montréal, 1977, 215p.

8. Marie-France Rochard, "Quellebibliothèque pour la recherche ? Enquête auprès des chercheurs scientifiques de 1'Université Claude Bernard Lyon In, Bulletin des bibliothèques de France, vol.29, no 1 (1984), 26-31.

9. Ibid., 30.

10. Mary B. Folster, «A Study of the Use of Information Sources by Social Science Researchers"), Journal of Academic Librarianship, vol.15, no.1 (March 1989), 7-11.

11. Martha Jane Soltow, «Public Libraries Service to Organized Labor: An Overview», R.Q, vol.24, no.2(1984), 163-168. 
À la FTQ, le centre de documentation est en voie d'informatisation. Les publications de la centrale seront, dans un premier temps, recensées dans la base de données. Les ouvrages et les publications répartis dans les différents bureaux des chercheurs seront ensuite centralisés au sein du centre de documentation. Ainsi, quand l'opération sera complétée, il sera possible de faire un inventaire exact du fonds documentaire.

Enfin, en plus de développer leur fonds documentaire, les centrales syndicales et leurs membres lèguent de plus en plus leurs fonds d'archives à des services d'archives $^{12}$.

\section{La provenance des demandes de re- cherche et la diffusion des résultats}

Quel que soit le syndicat national, les demandes de recherche proviennent le plus souvent des instances décisionnelles syndicales et les résultats de recherche reviennent à l'instance qui en a fait la demande.

\section{Fédération des travailleurs du Québec}

A la $\mathrm{FTQ}^{13}$, des demandes sont parfois acceptées venant des syndicats affiliés. Ces demandes sont ponctuelles et le type d'études qui en résultent est sectoriel. Par exemple, un syndicat implanté dans le secteur du meuble demande une recherche sur ce secteur de production. Si les chercheurs ne sont pas en mesure de répondre aux demandes de recherches, les syndicats se verront orientés vers d'autres groupes de recherche plus susceptibles de répondre à leurs besoins, comme par exemple, ceux du groupe régi par le Protocole UQAM-CSN-FTQ.

Les thèmes de recherche provenant des instances décisionnelles dépendent des priorités de la FTQ et des enjeux auxquels sont confrontés les travailleurs. Les orientations de la centrale sont votées en congrès. Le Conseil général, entre les congrès, actualise ces orientations et définit les actions de la centrale. Le Bureau gouverne entre les Conseils généraux. Les recherches ont comme principal objectif d'outiller la FTQ et ses syndicats affiliés pour qu'ils puissent mieux se dé- fendre lors des négociations collectives ou à l'occasion d'autres enjeux. Les recherches touchant à la conjoncture économique sont importantes: on pense à l'emploi, au chômage, etc. Un autre axe de recherche concerne les programmes fédéraux et provinciaux: la formation professionnelle, l'assurance-chômage, les juridictions relatives au monde du travail, etc.

Les résultats de recherche prennent des formes variées: mémoires, notes, brochures, dépliants, documents réalisés pour les colloques, documents de congrès, guide d'action, revues telles Le Monde ouvrier. Le plus souvent, les résultats de recherche sont destinés prioritairement aux membres de la FTQ. Parfois les recherches sont diffusées plus largement à l'extérieur, comme ce fut le cas pour le document de la Coalition intersyndicale contre le libreéchange, ou sous forme d'un mémoire. Pour faciliter l'accès et la diffusion des documents, une chercheure interrogée propose de regrouper les documents publiés par l'ensemble des organisations syndicales affiliées à la FTQ. L'idéal, selon elle, serait de regrouper les publications de toutes les organisations syndicales, populaires et communautaires dans une même base de données.

\section{Centrale des enseignants du Québec}

À la $\mathrm{CEQ}^{14}$, les grandes orientations de recherche sont discutées et votées lors des congrès généraux de la centrale. Le Conseil général reprend les décisions adoptées pour les synthétiser et les rendre disponibles à tous les membres. Ensuite, le Bureau national se charge d'élaborer des programmes d'action. Les demandes de recherche peuvent parfois venir des syndicats affiliés. Dans ce cas, l'aide que les chercheurs leur apportent se fait sous la forme de conseils plutôt que sous la forme d'études approfondies. Le service de recherche répond aussi aux demandes des coalitions qu'elles soient syndicales ou populaires.

Les publications prennent la forme de recensions, de recherches empiriques où les chercheurs se demandent comment les problèmes se traduisent dans les milieux de travail, de recommandations issues des congrès, de recherches utilisées lors des négociations et de recherches utilisées lors d'interventions politiques et publiques (par exemple, les mémoires). Dans le cas de recherches communes avec d'autres organismes, les résultats prennent plutôt la forme de rapport de réflexion.

Les documents de recherche sont généralement disponibles au public, sur demande. Les professeurs d'universités et les chercheurs d'autres groupes en sont notamment des utilisateurs. Ces documents sont recensés par le centre de documentation, (par exemple, la recherche de Jean-Claude Tardif intitulée L'évaluation en éducation. Certaines recherches plus approfondies sont publiées sous forme de monographies et distribuées à travers le réseau des librairies, tel l'ouvrage de Christian Payeur S'engager pour l'avenir (Québec, CEQ, 1990).

Pour permettre la diffusion des recherches effectuées à la CEQ, la centrale a créé une nouvelle revue qui se nomme Options. En plus de son rôle d'information, la revue «se veut un instrument de réflexion, de rayonnement et de démocratie» ${ }^{15}$. La CEQ semble se démarquer des deux autres syndicats en donnant plus d'importance à la communication de ses productions comme le montre la publication de sa nouvelle revue. La CEQ possède aussi un centre audiovisuel où elle produit des outils d'information et d'animation qui sont disponibles auprès des chercheurs ${ }^{16}$.

12. Le Service des archives de l'UQAM possède plusieurs fonds de syndicats et de militants syndicaux, notamment celui de la FTQ et de Émile Boudreau. Le Service des archives à l'Université de Montréal possède aussi des fonds d'archives syndicales, notamment ceux des syndicats de professeurs. Mentionnons le guide réalisé par JeanFrançois Cardin et Jacques Rouillard, Guide des archives des Unions internationales à Montréal, Montréal, Université de Montréal, FAS, Département d'histoire, 1987.

13. Les entrevues ont été réalisées le 15 mars 1991 dans les locaux de la FTQ à Montréal.

14. Les entrevues avec les chercheurs de la CEQ furent réalisées le 11 mars 1991 au service de recherche situé à Québec.

15. CEQ, Options, no 1 (printemps-été 1991), 6.

16. Par exemple, la CEQ a produit un vidéo sur la condition des Montagnais sur la Basse-Côte-Nord. 


\section{Confédération des syndicats nationaux}

Àla CSN17, les demandes proviennent des trois instances suivantes: la Confédération, les Fédérations et les Conseils centraux. $\AA$ chacune de ces instances correspondent des demandes de recherche particulières. Les Conseils centraux exigent du Service de recherche des analyses régionales tandis que les Fédérations demanderont des analyses sectorielles. Quantà la Confédération, les demandes se situent plutôt au niveau des activités politiques.

Les analyses comptent pour beaucoup dans le nombre des résultats de recherche publiés dans une année. En plus de ces analyses, le Service publie des guides syndicaux (par exemple, Le suivi de l'entreprise et La formation professionnelle). II diffuse, trois fois par année, un bulletin, Notes, publications et travail, qui répertorie les publications de recherche. Le Service de recherche n'a pas développé, au cours des années, de politique de publication. Un chercheur notait d'ailleurs, en entrevue, l'importance d'une telle politique qui pourrait définir les types de clientèles visés par les publications, le nombre et la fréquence de publications, etc. Un autre chercheur, d'autre part, mentionnait l'idée de publier un feuillet qui répertorierait les recherches effectuées dans le monde syndical québécois.

Finalement, les résultats de recherche peuvent prendre la forme d'exposé oraux lors de séances de formation aux responsables des Fédérations et des Conseils centraux de la CSN.

\section{Les recherches communes}

Chaque service de recherche étudié travaille sur des thèmes spécifiques à la centraleà laquelle il appartient. Par exemple, la FTQ, qui est fortement implantée dans l'industrie de la construction, ne produira pas les mêmes recherches que la $C E Q$ qui est composée majoritairement de personnel enseignant. Par ailleurs, sur d'autres sujets, comme la précarité d'emploi, chaque service produira des documents adaptés aux besoins spécifiques de ses membres.

Chaque centrale, de par sa composition, traite certains thèmes par des biais et des postulats propres à son idéologie. Un chercheur précisait que cela constitue une des raisons pour lesquelles il est difficile de tendre vers une plus grande intégration des services de recherches syndicaux.

Les recherches communes demeurent néanmoins appréciables. Mentionnons notamment les textes intersyndicaux concernant le libre-échange et les recherches réalisées dans le cadre du Comité conjoint UQAM-CSN-FTQ ${ }^{18}$. Ces recherches communes, nous semble-t-il, sont justifiées parce que les travailleurs affiliés dans des syndicats différents vivent souvent dans les mêmes situations, qu'il s'agisse de santé et sécurité au travail, de problèmes liés à la retraite, etc.

La réalisation des recherches communes, ou de plusieurs recherches tournant autour d'un même sujet, nous amène à nous questionner sur l'échange des informations et la mise en commun des ressources documentaires des centrales. Nous avons observé que les recherches communes ne suscitent pas de réflexion entraînant le développement d'échanges d'informations ni la mise en commun des ressources documentaires. Pourtant les chercheurs sont désireux d'augmenter les échanges d'informations. Nous verrons plus loin les avantages d'une telle mise en commun pour les chercheurs qui réalisent des recherches communes ou sur un thème qui n'est pas propre à une centrale.

La diffusion des recherches syndicales a lieu surtout à l'intérieur des syndicats. Les recherches susceptibles d'être publicisées à l'extérieur des syndicats sont les mémoires et les prises de positions sur des enjeux nationaux. Mais comme le manque de temps semble une contrainte importante commune à tous les chercheurs, les recherches de plus grande envergure semblent être fréquemment délaissées en faveur des demandes ponctuelles, peut-être moins intéressantes à publier.

On peut donc constater que les syndicats se prononcent sur un très grand nombre de questions, que ce soit seuls ou en collaboration, sans pour autant augmenter les ressources documentaires ou faciliter l'accès à ces ressources.
L'utilisation des sources documentaires

Les documents produits par la FTQ et ses syndicats affiliés (rapports, journaux syndicaux, etc.) constituent une première source de consultation pour les chercheures de la FTQ. Les publications syndicales du Québec, du Canada et d'autres pays constituent une autre mine d'informations. Les publications du Congrès du travail du Canada (CTC) en sont un exemple. Les publications des instituts de recherche sont également fort utiles ${ }^{19}$. Les documents statistiques sont également largement utilisés. L'une des chercheures travaille beaucoup avec les dossiers thématiques et les rapports issus des autres organisations. Les revues de presse sont importantes. Les publications officielles et les monographies sont, par contre, peu utilisées. II demeure que les documents privilégiés contiennent des informations factuelles dans lesquels les chercheures trouveront des éléments pour permettre à la FTQ d'agir et de solutionner des problèmes pratiques. Ces documents permettent aux chercheures de se nourrir des expériences des autres syndicats (au Québec et dans les autres pays) pour mieux intervenir localement ou nationalement.

Quant aux bibliothèques et services de documentation extérieurs au centre de documentation, les chercheures de la FTQ les utilisent relativement peu. Leurs besoins actuels n'exigent pas une fréquentation régulière d'autres bibliothèques. Deux ou trois fois par mois, une chercheure consulte les ouvrages à la bibliothèque des Hautes études commerciales (HEC), au centre de documentation de Statistique Canada, à la Bibliothèque des Lettres et

17. Les entrevues ont été réalisées en mars 1991 dans les locaux de la CSN à Montréal.

18. Pour n'en nommer que deux, mentionnons: Comité conjoint UQAM-CSN-FTQ, Les radiations ionisantes en milieu de travail, 1990, 209p. et L'essayer c'est l'adopter - Les obstacles ergonomiques à l'accès des femmes aux postes traditionnellement masculins, 1990, 48p.

19. Les instituts de recherche sur le travail sont nombreux. Mentionnons deux organismes de recherche qui sont en France: l'IRES (Institut de recherches économiques et sociales) et l'ANACT (Agence nationale d'amélioration des conditions de travail). 
Sciences humaines de l'Université de Montréal (BLSH) et à l'IRAT.

Les chercheures de la FTQ consacrent néanmoins très peu de temps à la recherche documentaire. Le service de recherche est petit et le centre de documentation n'est pas abonné à des serveurs de bases de données. On peut émettre l'hypothèse que les chercheures augmenteront sensiblement le temps consacré à la recherche documentaire lorsque la base de données deviendra fonctionnelle et qu'elles auront accès à des serveurs.

À la CEQ, les chercheurs confient le travail de recherche bibliographique aux bibliothécaires du centre de documentation. Les chercheurs se libèrent ainsi d'un temps précieux pour mieux réaliser leurs recherches. Lorsqu'un état de la question est nécessaire, les chercheurs font appel à des étudiants-chercheurs de deuxième ou de troisième cycle. Par exemple, dans le cadre d'une recherche qui a duré un an, un chercheur a engagé deux personnes pour faire une revue de la littérature assortie d'une recherche bibliographique.

Les deux chercheurs de la CEQ disposent d'une période de temps plus grande que les chercheurs des deux autres centrales pour réaliser leurs recherches, même s'ils considèrent être pressés par le temps. Pour eux, les documents produits par la $C E Q$ sont jugés très importants. Les rapports, les dossiers et les revues le sont moins. Les journaux, les statistiques et les publications officielles viennent en fin de liste. Un chercheur a également soulevé l'idée de répertorier les publications des groupes communautaires et populaires. Selon lui, on sous-estime l'intérêt de ces publications. En sachant ce qui s'écrit dans les milieux communautaires et populaires, les chercheurs syndicaux pourraient éviter parfois d'entamer des recherches déjà effectuées. Ils pourraient aussi s'alimenter des sujets de recherches et les pousser plus loin dans la réflexion. Les groupes communautaires et populaires n'ont pas les ressources pour effectuer des recherches approfondies.

À la $C E Q$, les ressources du centre de documentation étant relativement abondantes, les besoins d'aller consulter à l'extérieur sont moins grands.

C'est à la CSN que la recherche documentaire occupe le plus de temps du travail des chercheurs. Cela tient au fait qu'ils font eux-mêmes leurs recherches bibliographiques. Certains chercheurs consacrent donc moins de temps qu'ils le désirent à leur travail de recherche proprement dit. Tous les types de documents sont dignes d'attention, mais certains le sont particulièrement, commeles publications officielles, les ouvrages statistiques et les dossiers.

Les chercheurs de la CSN fréquentent au besoin les autres bibliothèques dans le cas où ils ne peuvent trouver certains documents essentiels à leur centre de documentation ou qu'ils ne peuvent les faire venir par des prêts entre bibliothèques. Les bibliothèques fréquentées sont la bibliothèque des HEC et le centre de documentation de Statistique Canada. Selon un chercheur, la visite des centres de documentation extérieurs est moins fréquente qu'auparavant. Le centre de documentation est aujourd'hui plus riche et plus fonctionnel. En outre, depuis l'avènement des bases de données, les chercheurs n'ont plus à se déplacer autant qu'avant.

\section{Les sources orales d'information}

Comme dans tout milieu de recherche spécialisé, les sources orales d'informations sont capitales dans le mouvement syndical. Un chercheur de la CSN en voyait deux types: le réseau interne, c'està-dire toutes les personnes qui possèdent les informations essentielles au travail de recherche entrepris (chercheurs, responsables syndicaux, etc.); les rencontres que l'on fait avec les travailleurs directement impliqués par le sujet de recherche. Ces travailleurs parlent de ce qu'ils connaissent et de ce qu'ils espèrent comme améliorations futures. Ces rencontres permettent de brosser un premier portrait de la situation et de commencer la recherche. Elles sont essentielles, car cesont les travailleurs qui bénéficieront des conclusions. Un chercheur de la CSN a utilisé l'appellation de «recherche utilitaire». Ces recherches ont pour but de conseiller les gens impliqués dans les milieux et doivent déboucher sur des outils concrets de changement.

Pour un chercheur de la CEQ, les sources orales permettent de prendre con- naissance des sujets de recherche nouvellement abordés par d'autres chercheurs.

Les informations orales recueillies valident ou invalident les résultats de recherche. Selon une chercheure de la FTQ, «elles apportent l'aspect qualitatif à nos analyses qui sont plus de l'ordre du quantitatif. Pour cela, dit-elle, il est nécessaire et important d'appeler nos affiliés, de les rencontrer pour qu'ils puissent nous nourrir de leur vécu».

Quand il s'agit d'échanger des informations entre les services de recherche des centrales, les sources orales sont les plus utilisées. Les chercheurs des différents syndicats se téléphonent, se rencontrent, s'échangent de l'information. Mais le tout ne se fait pas dans un cadre formel. Protocole UQAM-FTQ-CSN et I'IRAT jouent un rôle de collaboration entre les chercheurs.

\section{L'utilisation des bases de données}

Les bases de données sont venues modifier grandement les habitudes documentaires des chercheurs. Elles leur font économiser beaucoup de temps. Une interrogation en ligne chez un serveur assure l'acquisition immédiate des informations factuelles et bibliographiques les plus récentes.

À la CSN, ce sont les chercheurs euxmêmes qui interrogent les bases de données. Toutefois, tous ne sont pas habilités aux langages d'interrogation, même s'ils deviennent de plus en plus familiers avec les bases de données. Ils sont, par contre, tous convaincus de la pertinence de celles-ci. Un chercheur a dit interroger les banques quatre ou cinq fois par semaine, mais il demeurait, par ailleurs, plus habile à manipuler les sources écrites. Selon lui, les chercheurs réalisent des économies de temps en utilisant les bases de données. De plus, elles sont mises à jour plus régulièrement que les sources écrites.

Les bases de données, tant bibliographiques que factuelles sont jugées essentielles à la CSN. Certains chercheurs, par exemple, spécialisés dans le traitement des statistiques travaillent dans le cadre d'une recherche sur la situation 
économique des entreprises. Ils consultent obligatoirement les bases de données financières et statistiques (STATCAN). Pour le coordonnateur du service de recherche de la CSN, les bases de données sont devenues indispensables. II travaille d'ailleurs à l'élaboration de mini-guides pour aider les chercheurs à connaître les différents langages d'interrogation ainsi qu'à maîtriser les principales fonctions.

Par ailleurs, les bases de données ne répondent pas entièrement aux besoins des chercheurs. Le plus ou moins grand recours aux bases de données dépend en partie de la nature des recherches. Par exemple, les bases de données seront moins utiles pour les recherches de très courte durée.

Àla FTQ, on ne retrouve pas encore de bases de données. Comment les chercheures perçoivent-elles la venue prochaine des bases de données dans les services de recherche? Les avis sont partagés. D'une part, la recherche n'étant pas assez poussée à la FTQ, une chercheure ne voit pas l'urgence de s'y abonner. Une autre chercheure partage un point de vue différent. Elle est d'avis que les bases de données seraient utiles car elles donnent accès, et rapidement, à des informations spécialisées et factuelles comme les indices des prix à la consommation, les statistiques sur la pauvreté, etc., autant de données pertinentes à ses recherches. La chercheure estime qu'avec la consultation de certaines bases de données, le centre de documentation pourrait même cesser certains de ses abonnements.

À la CEQ, les chercheurs font faire leurs recherches en ligne par les bibliothécaires et documentalistes. L'interrogation des bases de données constitue pour les chercheurs la première étape avant d'entreprendre la recherche. Les ouvrages importants tirés des listes bibliographiques seront achetés ou empruntés (PEB) par le centre de documentation.

\section{Conclusion}

En résumé, dans tous les syndicats, les demandes en matière de recherche proviennent des instances décisionnelles. Les résultats de recherche prennent sensible- ment les mêmes formes d'une centrale à l'autre. Les recherches sont diffusées surtout à l'intérieur des syndicats pour répondre aux besoins des membres. Des trois centrales, la CEQ est celle qui porte le plus grand intérêt à la diffusion extérieure.

Les types de recherche et les thèmes abordés varient en fonction de la composition des centrales et des priorités de celles-ci.

La FTQ se démarque des deux autres centrales en faisant un type de recherche moins approfondie, plus pratique et plus axée sur les solutions à apporter à un problème. C'est à la CEQ que les recherches de longue haleine se font le plus souvent. Le service de recherche est d'ailleurs supporté par le centre de documentation. Les chercheurs travaillent continuellement et conjointement avec les documentalistes. La CSN regroupe en son sein des travailleurs de milieux divers (professionnels, employés de soutien, enseignants, etc.). Les chercheurs doivent donc répondre à des demandes plus hétérogènes. Un problème demeure irrésolu à la CSN: la documentation est répartie entre le service de recherche et le centre de documentation.

Les besoins documentaires ne varient pas grandement d'un chercheur à l'autre. Les attentes de l'ensemble des chercheurs envers les services fournis par les centres de documentation se résument ainsi: les centres doivent fournir des service de support à la recherche, ils doivent fournir rapidementl'information demandée et prévoir les besoins des chercheurs.

Les sources orales d'information et les bases de données (exception faite de la FTQ qui n'est pas abonnée aux bases de données) sont importantes pour la majorité des chercheurs.

L'informatisation des centres de documentation à la CSN et à la CEQ a ouvert de nouvelles avenues. Elle a permis un repérage plus rapide et plus efficace de l'information. Avec l'informatisation prochaine du centre de documentation de la FTQ, d'autres possibilités de collaboration s'offrent aux centres de documentation, tel que l'échange des bases de données entre les centres. L'exemple de la CEQ nous montre les possibilités, encore peu exploitées, d'échanger de l'information et d'avoir un accès en ligne à une base de données. Des chercheurs ont exprimé le souhait de multiplier ces échanges d'information. Ils ont, notamment, exprimé l'idée de voir rassemblé dans une seule base de données l'ensemble des publications produites par tous les syndicats. D'autres ont émis des réserves à l'échange et par conséquent à la mise en commun de l'information.

Les avantages les plus évidents d'une collaboration plus étroite, entre les centres de documentation, sont les suivants: la connaissance des autres centres de documentation, la possibilité d'accélérer l'accès aux documents (avec le PEB, par exemple), et l'échange réciproque de services. Actuellement, chacun ne saitpas ce que l'autre possède. La mise en commun, totale ou partielle, des ressources par le partage des catalogues (échange de listes imprimées des catalogues, échange de listes sur disquettes, etc.) pourrait éviter que chacun se coupe d'une masse d'informations pertinentes à la portée de la main. Cette plus grande collaboration entre chacun pourrait éviter la redondance dans l'achat de documents.

Ceci dit, des échanges d'information ont quand même lieu. Ils se font souvent dans un cadre informel. La prochaine étape, donc, serait de recourir aux possibilités de l'informatisation pour mieux répondre à la recherche syndicale, en permettant aux chercheurs de mieux réaliser leur mandat et en améliorant les services que les chercheurs rendent à la centrale et aux syndiqués. 